\title{
Contribution of perceptual motor dysfunction to construction and tracing disturbances in Parkinson's
} disease

\author{
YAAKOV STERN,${ }^{*}+$ RICHARD MAYEUX,${ }^{*}$ JEFFREY ROSEN $\dagger$ \\ From the Departments of Neurology, Columbia University, College of Physicians and Surgeons, ${ }^{*}$ and \\ Psychology, City University of the City of New York, $\dagger$ USA
}

SUMMARY Patients with Parkinson's disease and controls traced patterns of increasing complexity presented on a vertical transparent screen. Some patterns were presented with missing segments which subjects were required to fill in. Digitising equipment quantitated movements for computer analysis. Subjects also completed a brief test of general intellectual function, a construction test and an assessment for depression, and the severity of patients' Parkinsonian signs and symptoms was rated. Patients performed more poorly than controls on complete patterns, and their errors increased more sharply than controls on patterns with missing segments. Patients' errors, but not those of controls, in filling in missing segments were uniquely related to performance on construction tasks. Other aspects of patients' tracing performance correlated with the severity of their Parkinsonian symptoms. These findings suggest that there is an underlying perceptual motor deficit in Parkinson's disease that affects performance on both tracing and construction tasks.

In a previous study, ${ }^{1}$ perceptual motor dysfunction in Parkinson's disease was explored using a task in which patients traced patterns and then filled in missing sections of those patterns. A relationship between tracing performance and scores on tests of construction and general intelligence was noted. In that study, videotaped tracing performance was rated. Using this method, the specificity of the relationship to particular intellectual functions was not established. Certain aspects of tracing performance seemed to be related to the motor manifestations of the disease, but we could not demonstrate this relationship. Also, the different errors made by Parkinsonians and controls when tracing paths with missing segments were not established quantitatively because our rating system focussed on qualitative aspects of tracing rather than quantifying movements. In order to re-examine these issues, we tested patients and controls on a similar tracing task,

Presented in part at the 1983 meeting of the Society of Neuroscience, Boston, Mass, USA

Address for reprint requests: Dr Y Stern, Department of Neurology, 710 W. 168th Street, New York, N. Y. 10032, USA.

Received 9 September 1983 and in revised forn. 5 March 1984 Accepted 10 March 1984 using digitising apparatus to quantify their movement speed and accuracy. Using this new approach, more error by patients than controls was noted as segments of paths were deleted, a specific relation of tracing errors to construction performance in the Parkinsonian patients was found, and a correlation between other aspects of patients' tracing performance and ratings of the motor manifestations of the disease was established. These findings aid in developing a clearer understanding of perceptual motor dysfunction in Parkinson's disease.

\section{Methods}

\section{Subjects}

Sixteen patients with idiopathic Parkinson's disease were tested. Medication for their symptoms was unchanged over two months before entry. Nine subjects with no history or signs of neurologic or psychiatric disorder served as controls. None of the subjects had been studied previously. All subjects were volunteers and gave informed consent; none were on medications that affect intellectual function, or had a history or signs of dementia as determined by neurological or neuropyschological evaluation. All but one subject were right handed. A summary of demographic characteristics of subjects is given in table 1 .

\section{Procedure}

All subjects were examined by the same neurologist, who 
Table 1 Demographic characteristics, neuropsychological test scores and Parkinson's disease symptom severity scores of subjects (Values in parentheses are standard deviations, $p$ values are for $t$ test comparisons)

\begin{tabular}{llll}
\hline & $\begin{array}{l}\text { Parkinson's disease } \\
(N=16)\end{array}$ & $\begin{array}{l}\text { Controls } \\
(N=9)\end{array}$ & $p$ \\
\hline Age & $60.6(10.4)$ & $67.0(3.2)$ & .05 \\
Education & $13.2(2.5)$ & $11.2(1.1)$ & .01 \\
Mini Mental State & $50.7(5.1)$ & $53.2(2.0)$ & n.s. \\
$\begin{array}{l}\text { Rosen Drawing } \\
\text { Beck Depression }\end{array}$ & $11.1(2.4)$ & $12.5(1.7)$ & n.s. \\
$\quad \begin{array}{l}\text { Inventory } \\
\text { Parkinson's disease }\end{array}$ & $7.9(6.9)$ & $3.8(2.9)$ & n.s. \\
$\begin{array}{l}\text { Evaluation } \\
\text { Tremor }\end{array}$ & $30.9(4.8)$ & & \\
Rigidity & $1.8(2.1)$ & & \\
Bradykinesia & $4.4(2.8)$ & & \\
Duration of Illness & $1.4(1.0)$ & & \\
\hline
\end{tabular}

had no knowledge of subjects' performance on other aspects of the study. For the patients, the Columbia University Parkinson Disease Evaluation ${ }^{2}$ was used to rate signs and symptoms of Parkinson's disease. Each sign and symptom was rated according to defined criteria from 0 to $4(0=$ absence; $4=$ greatest severity $)$. Rigidity and tremor ratings were the sum of ratings of each of the four limbs and the neck or head. Mean Parkinson's disease scores and duration of illness are included in table 1.

The Beck Depression Inventory (BDI) was used to rate depression $^{3}$ (table 1). This is a self report instrument in which the subject, in the presence of the examiner, denies or affirms to various degrees 21 statements associated with symptoms of depression.

The Mini-mental Status Examination (MMS) ${ }^{4}$ as modified by Mayeux et al..$^{5}$ was administered (table 1). This is divided into subtests representing different aspects of intellectual function. Maximum score is 57 . In addition, the Rosen Drawing Test $^{6}$ was administered (table 1). In this test the subjects copies 15 designs of increasing complexity. Designs were selected to test different levels of spatial representation ranging from topographic to Euclidean to perspective geometries.

The tracing task was similar to that used in our earlier study.' The apparatus consisted of a clear screen ( 3 feet $X$ 3 feet) mounted perpendicular to a testing table. Paths drawn on clear plastic were affixed to the screen (fig 1). The lines of the paths were $0.79 \mathrm{~mm}$ wide. Path 1 was a straight line, and Path 2 consisted of two endpoints which the subject connected in a straight line. Paths 3-6 were the same sawtooth pattern, but Paths 4-6 had progressively increasing segments deleted. The subject traced the paths with a stylus. The subject was presented with patterns in the sequence shownin Figure 1 and instructed to trace the pattern with the stylus beginning from the right side and, at the end of the pattern, return to the starting point. Left hand performance followed right. On patterns with deleted segments, the subject was instructed to attempt to complete the pattern by filling in the missing segments while tracing.

Mounted on the tracing screen was the "L" shaped microphone array of a sonic digitiser (Scientific Accessories Corporation GP6-25H). This microphone array and the stylus that was moved along the paths during tracing were

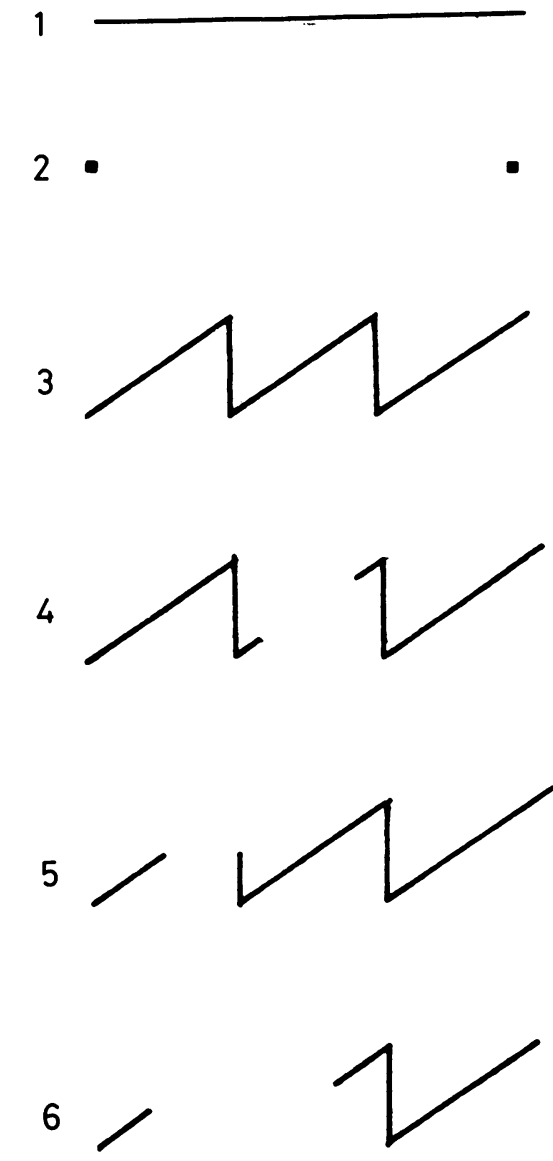

Fig 1 Paths used in tracing task in order of their administration.

connected to the digitising unit. The stylus emitted a series of sounds that were picked up by the microphone array and transferred to the digitising unit, which then converted this sonic information into a Cartesian, $X-Y$ coordinate describing the position of the stylus at an accuracy within $0.01 \mathrm{~cm}$. Coordinates were generated approximately 100 times per second, transmitted for storage to a digital disc recorder (Sykes ComStor 1), and later transferred to a computer (Digital MINC-11) for off-line analysis.

Two new aspects of tracing performance, tracing accuracy and velocity, were quantified. Tracing accuracy was measured as area error. The stylus position at each point was compared with the position of the closest point on the pattern. Tracing movement coordinates and the intersected coordinates of the path were treated as if they outlined a series of irregular polygons, and the area of these polygons was calculated.

Since points were digitised at a regular rate (100 points/ s) the velocity measure was simply the number of points digitised during the tracing of a specific extent of a path. The number of digitised points increased with tracing time. 
Tracing measures (area error and digitised points) were calculated separately for both sweeps of each path and for the right and left hand. A sweep consisted of the tracing of a path in one direction.

\section{Results}

Parkinsonians were younger and better educated than controls (table 1). Since age and education affect performance on cognitive tests, ${ }^{5}$ it would be expected that the modified MMS and Rosen Drawing Test scores would be higher in the Parkinson's disease group. However, scores on these tests were comparable in the two groups. It is possible that if controls and Parkinsonians were more closely matched for age and education, controls would have performed better on the modified MMS and Rosen. Alternatively, it is useful to have the groups matched for performance on these tasks in order to demonstrate relative deficits in the tracing task in the face of comparable performance on these paper and pencil tasks.

Since depression is common in Parkinson's disease and might affect intellectual performance, ${ }^{5}$ it was important to compare the severity of depression in the two groups. While the mean Beck Depression Inventory score was slightly higher in the Parkinson's disease group, the scores did not differ (table 1). It can be assumed that depression did not play a major role in intergroup differences in performance.

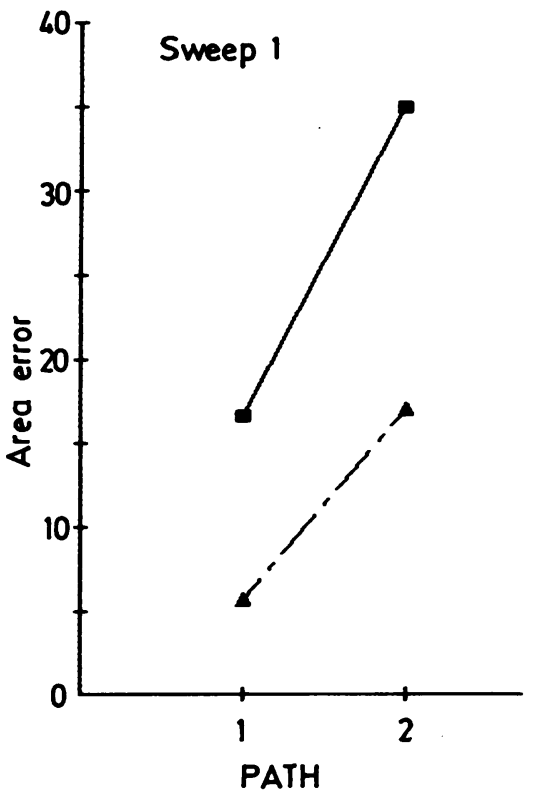

Comparison of tracing performance in Parkinson's disease and control groups

Differences between Parkinson's disease and control group performance on the tracing task were analysed separately in both sweeps of straight-line (Paths 1-2) and sawtooth paths (Paths 3-6). In each case, repeated measure ANOVA's evaluated changes in each tracing measure as segments of the paths were deleted. Effects in the ANOVA included Group (Parkinson's disease vs. control), Path (for example, Path 1 vs. 2), Hand (Right vs. Left), and the appropriate interactions of these effects.

Straight-line paths. In the analysis of the area error measure in sweep 1 of the straight-line paths (Paths $1-2)$, significant effects included Group $(p<0.01)$, Path $(\mathrm{p}<0.01)$, and the Group X Path interaction $(p<0.05)$ (fig 2). The group effect indicated poorer overall performance in the Parkinson's disease group. While performance was poorer on Path 2 than Path 1 in both groups, the decrement in performance was greater in the Parkinson's disease group. This accounted for the significant interaction effect. In sweep 2, Group and Path effects were significant $(p<0.01)$, but not the interaction effect. The Group effect indicated poorer performance by the Parkinson's disease group. The Path effect indicates that combined patient and control performance was worse in Path 2.

For the velocity measure (digitised points), there were significant Group, Path, and Hand effects in

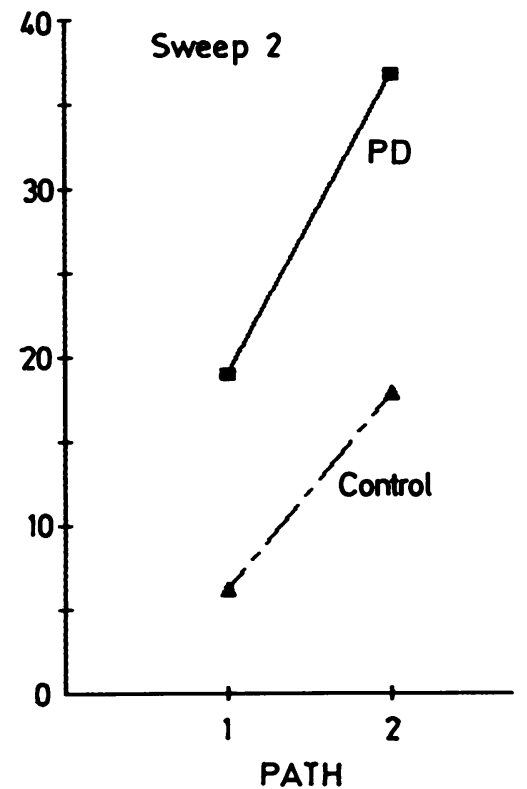

Fig 2 Area error in Paths 1 and 2. 
sweep $1(p<0.05)$, indicating more rapid tracing in the Parkinson's disease group, on Path 2, and with the right hand respectively. In sweep 2 , only the Path effect was significant $(p<0 \cdot 01)$, indicating faster tracing by both groups on Path 2 .

Sawtooth paths. A second analysis examined changes in tracing performance in the sawtooth patterns as segments were deleted. Observations in pilot work suggested that in addition to affecting tracing in the deleted segments, performance on the remainder of the path was also influenced. This occurred for various reasons: (1) subjects adjusted or slowed their tracing movements before entering the missing segments, (2) subjects paused prior to beginning to trace the missing segments, (3) when subjects concentrated on filling in missing segments performance on remaining portions was not as carefully executed, and (4) when filling in missing segments incorrectly, subjects often could not rejoin the path in a way that would allow them continue tracing it accurately. While none of these events occurred consistently, they did suggest that if performance were evaluated only in the deleted segment, some information about the effects of the deleted segments on tracing performance could be missed. Therefore, the analysis compared the tracing performance measures over entire sweeps of Paths 3-6.

Area error in Paths 3-6 is illustrated in fig 3. In sweep 1, significant effects included Group ( $p<$ $0.01)$, Path ( $<<0.01)$, Hand $(p<0.05)$, and the
Group $\times$ Path interaction $(p<0 \cdot 01)$. The Group $\times$ Path interaction was due to an increase in area error in the Parkinson's disease group from Paths 3 to 6 that was greater than in the control group. In addition, patients' overall performance was poorer than controls', and all subjects performed better on the earlier paths and with their right hand. In sweep 2, Group, Path, Hand, and Group $\times$ Path $\times$ Hand effects were significant ( $p<0.01$ for all effects). Area error increased in the Parkinson's disease group from Path 5 to 6 in both hands, but in the control group an increase was seen only in the right hand. Overall, patients performed more poorly than controls, and all subjects performed on the earlier paths and with their right hand.

Significant Group $\times$ Path interactions were seen in both the straight-line and in sawtooth paths. This suggested a specific deficit in the Parkinson's disease patients for tracing accuracy in paths with deleted segments.

Analysis of digitised points in the sawtooth paths revealed no significant effects in sweep 1 , but there were significant Path $(p<0.05)$ and Hand $(p<$ 0.01 ) effects in sweep 2 . These indicated slower tracing with left than the right hand, and a tendency for subjects to trace faster in the later paths.

Relation of tracing performance to cognitive tests and demographic variables

The relationship of tracing measures to performance on the modified MMS and Rosen Drawing Test, as well as to the age and education of the subjects, was
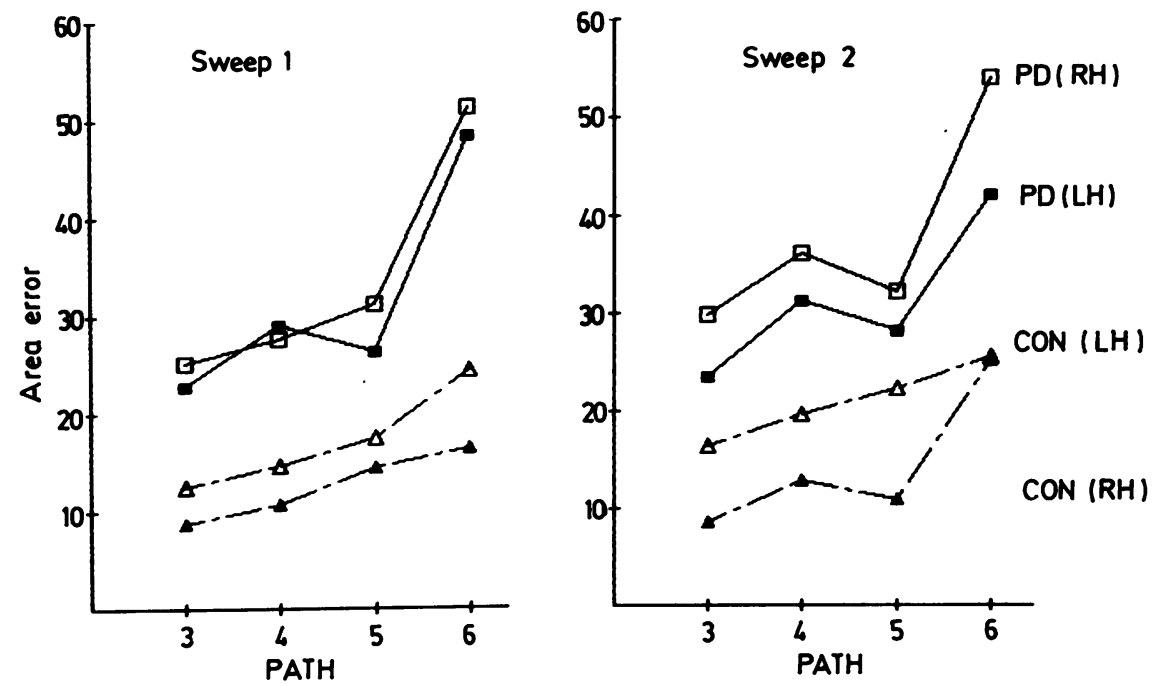

Fig 3 Area error in Paths 3 through $6(\mathrm{RH}=$ Right Hand; $L H=$ Left Hand; CON = Control). 
Table 2 Relation of tracing performance in Parkinson's disease patients to measures of Parkinson's disease severity

\begin{tabular}{|c|c|c|c|c|c|}
\hline Path & Sweep & Tracing Variable & Related Variables & $r$ & $p$ \\
\hline $\begin{array}{l}1 \\
1 \\
1\end{array}$ & $\begin{array}{l}1 \\
1 \\
2 \\
2 \\
2 \\
2 \\
1 \\
2 \\
2 \\
2\end{array}$ & $\begin{array}{l}\text { Digitised points } \\
\text { Digitised points } \\
\\
\text { Digitised points } \\
\text { Area error } \\
\text { Digitised points } \\
\text { Area error }\end{array}$ & $\begin{array}{l}\text { Duration of illness } \\
\text { Bradykinesia } \\
\text { Parkinson's disease severity } \\
\text { Rigidity } \\
\text { Bradykinesia } \\
\text { Postural stability } \\
\text { Rigidity } \\
\text { Bradykinesia } \\
\text { Rigidity } \\
\text { Tremor }\end{array}$ & $\begin{array}{l}0.44 \\
0.416 \\
0.528 \\
0.608 \\
0.607 \\
0.433 \\
0.423 \\
0.479 \\
0.533 \\
0.425\end{array}$ & $\begin{array}{l}0.05 \\
0.05 \\
0.01 \\
0.01 \\
0.01 \\
0.05 \\
0.05 \\
0.05 \\
0.01 \\
0.05\end{array}$ \\
\hline
\end{tabular}

assessed. Before performing these analyses, tracing measures were compared for differences between performance on the first and second sweep or left and right hand using $t$-tests for repeated measures. There was no significant difference between right and left hand performance on any path. However, there were significant differences between performance on the first and second sweeps on almost every path. Therefore, left and right hand performance was combined, while data from first and second sweeps were analysed separately.

In the control group, tracing performance correlated with subtests of the modified MMS that measured general intelligence, age, level of education, and depression ratings, but not with the construction subtest of the modified MMS or the Rosen Drawing Test. No clear pattern of relationship between tracing performance and specific cognitive functions could be discerned; performance seemed to relate more to general intelligence. In the Parkinsonian group, a similar pattern prevailed for Paths 1-4. However, in Paths 5 and 6 measures of tracing performance correlated almost exclusively and consistently with performance on the construction subtest of the modified MMS and the Rosen Drawing Test.

Correlations were also calculated between measures of Parkinson's disease severity and tracing performance in the Parkinson's disease group (table 2). Significant correlations were found primarily in the straight-line paths (Paths 1 and 2), and Parkinsonian symptoms generally correlated with tracing velocity. It was not possible to isolate a clear pattern of interrelationships between specific aspects of tracing performance and specific measures of Parkinson's disease symptom severity.

\section{Discussion}

Patients' tracing accuracy was poorer than controls', and declined even more than the controls' on paths with deleted segments. This effect was demonstrated statistically in two important contrasts, the comparison of area error on the straight-line paths (Paths 1-2), and on the sawtooth paths (Paths 3-6). The most stringent general interpretation of these findings is that the motor manifestations of Parkinson's disease accounted for the generally lowered tracing accuracy of patients on all paths, while the additional decline in performance on paths with deleted segments was due to some intellectual deficit, or problem of "higher-order" motor control.

The consistent and unique relationship of Parkinsonians' tracing error on the more difficult paths with missing segments (Paths 5 and 6) to performance on construction tests suggests that the tracing and construction tasks tapped a similar cognitive process. In contrast, tracing error in controls correlated with indices of general intelligence and not with construction. In our earlier study' we had noted a relationship between tracing, construction, and intellectual function in Parkinson's disease, but the quantitative measures in this study allowed us to isolate a specific relation to construction.

Another factor that correlated with patients' tracing performance was the severity of the motor manifestations of Parkinson's disease. In our previous study, the effect of these symptoms was inferred, but no direct correlations were observed. It was not possible in this study to isolate particular symptoms that affected performance, but tracing velocity was most frequently related to motor symptom severity.

Deficits in Parkinson's disease are commonly noted on construction, ${ }^{7-10}$ orientation, ${ }^{10-13}$ and tactile tacks. ${ }^{14}$ Tracing ${ }^{1}$ and tracking ${ }^{15-17}$ deficits have often been noted, as well as difficulties in sequencing simultaneous movements. ${ }^{18} 19$ Flowers ${ }^{17}$ and others ${ }^{1520}$ have called attention to deficits in predictive movement, that is, movement that takes place with either no external guidance or too rapidly to monitor continuously. Similar deficits, such as in tracking $^{2122}$ and orientation ${ }^{2324}$ have been reported in animals with caudate lesions, implicating the basal ganglia in these processes.

In this study, poor performance in filling in missing segments of paths could be considered a deficit in sequential and predictive movement. Patients either could not generate a proper motor plan to guide the movements or could not carry out that 
plan. The severity of this deficit was related to performance on two construction tasks. Construction deficits can also stem from an ability to plan constructional activity or to check the accuracy of that activity. ${ }^{25}$ Therefore, these data suggest that a general deficit in Parkinson's disease that we have sug- gested be called perceptual motor dysfunction. ${ }^{26}$ This term implies that difficulty lies in coordinating the motor and perceptual activities necessary to successfully carry out these tasks. This inability is particularly apparent as external guidance from the environment is removed, for example, as segments in traced paths are deleted. Deficits in the sequencing of simultaneous movement ${ }^{18} 19$ or in performing rapid movements ${ }^{16}$ also suggest a problem in controlling these measurements when the activity cannot be continuously monitored.

Perceptual motor dysfunction in Parkinson's disease may apply only to tasks that demand motor output, but the dysfunction as outlined could apply to purely cognitive tasks as well. In the Wisconsin Card Sort, which patients with Parkinson's disease perform poorly, ${ }^{87}$ responses must be modulated based on the success of past responses. This may also reflect a perceptual motor dysfunction. ${ }^{28}$ More to the point are deficits in spatial tasks that demand no motor output. These imply that perceptual motor dysfunction affects not only motor control but the underlying coordination of sensory and motor activities that is necessary for the development of internal spatial representation. Deficits of this nature have been suggested in studies of spatial memory ${ }^{29}$ and multiple choice spatial tasks. ${ }^{30}$

In conclusion, deficits in perceptual motor function affect both construction and the control of sequential and predictive movement in Parkinson's disease and may also result in impaired internal spatial representation.

We thank the staff and members of the South East Queens Multi-Service Senior Citizens Center for their aid and participation in this study, and Drs Cote and Fahn for their assistance. This research was supported by the Parkinson's Disease Foundation (Dr Stern), and Federal Grant AG 02802 (Dr Mayeux).

\section{References}

' Stern Y, Mayeux, Rosen J, Ilson J. Perceptual motor dysfunction in Parkinson's disease: a deficit in sequential and predictive voluntary movement. J Neurol Neurosurg Psychiatry 1983;46:145-51.

${ }^{2}$ Lesser RP, Fahn S, Snider SR, Cote LJ, Isgren WP, Barrett RE. Analysis of clinical problems in parkinsonism and in the complications of long-term
Levodopa therapy. Neurology (Minneap) 1979; 29: $1253-60$.

${ }^{3}$ Beck AT, Ward CH, Mendelson M, Mock J, Erbaugh J. An inventory for measuring depression. Arch Gen Psychiatry 1961;4:53-61.

${ }^{4}$ Folstein MF, Folstein SE, McHugh PR. "Mini-mental State", A practical method for grading the cognitive state of patients for the clinician. J Psych Res 1975;12:189-98.

s Mayeux R, Stern Y, Rosen J, Leventhal J. Depression, intellectual impairment, and Parkinson's disease. Neurology (NY) 1981;31:645-50.

${ }^{6}$ Rosen W. The Rosen Drawing Test. Veterans Administration Medical Center, Bronx, New York, 1981.

' Loranger AW, Goodell H, McDowell FH, Lee JE, Sweet RD. Intellectual impairment in Parkinson's syndrome. Brain 1972;95:405-12.

${ }^{8}$ Yahr HD, Proctor-Bowen F. Intellectual deficits in parkinsonism. Int J Neurol 1975;10:280-1.

${ }^{9}$ Botez MI, Barbeau A. Neuropsychological findings in Parkinson's disease: A comparison between various tests during long-term Levodopa therapy. Int J Neurol 1975; 10:222-32.

10 Joubert M, Barbeau A. Akinesia in Parkinson's disease. In: Barbeau A, Brunette JR, eds. Progress in Neurogenetics. Amsterdam: Excerpta Medica, 1969:366-76.

" Bowen FP. Behavioral alterations in patients with basal ganglia lesions. In: Yahr MD, ed. The Basal Ganglia. New York: Raven Press, 1976:169-80.

12 Danta G, Hilton RC. Judgment of the visual vertical and horizontal in patients with parkinsonism. Neurology (Minneap) 1975;25:43-7.

${ }^{13}$ Teuber H-L, Proctor F. Some effects of basal ganglia lesions in subhuman primates and man. Neuropsychologia 1964;2:85-93.

${ }^{14}$ Mayeux R, Tomaino C, Rosen J, Stern Y, Gerstman L. The effects of parkinsonism and aging on tactilemotor skills and perception. Neurology (NY) 1983;33:A198.

15 Draper IT, Johns JJ. The disordered movement in parkinsonism and the effect of drug treatment. Bull John Hopkins 1964;115:465-80.

${ }^{16}$ Flowers K. Some frequency response characteristics of parkinsonism on pursuit tracking. Brain 1978;101:19-34.

${ }^{17}$ Flowers K. Lack of prediction in the motor behaviour of parkinsonism. Brain 1978;101:35-52.

${ }^{18}$ Perret E. Simple motor performance of patients with Parkinson's disease before and after a surgical lesion in the thalamus. J Neurol Neurosurg Psychiatry 1968;31:284-90.

${ }^{19}$ Schwab RS, Chafetz ME, Walker S. Control of two simultaneous voluntary motor acts in normals and in parkinsonism. Arch Neurol Psychiat 1954;75:591-8.

${ }^{20}$ Cooke JD, Brown JD, Brooks VB. Increased dependence on visual information for movement control in patients with Parkinson's disease. Can J Neurol Sci 1978;5:413-5.

${ }^{21}$ Bowen FP. Visuomotor deficits produced by cryogenic lesions of the caudate. Neuropsychologia 1969;7:5965. 
${ }^{22}$ Hore J, Meyer-Lohmann J, Brooks VB. Basal ganglia cooling disables learned arm movements of monkeys in the absence of visual guidance. Science 1977; 195:584-6.

${ }^{23}$ Divac I. Neostriatum and functions of the prefrontal cortex. Acta Neurobiol Exp 1972;32:461-77.

${ }^{24}$ Potegal M. The caudate nucleus egocentric localization system. Acta Neurobiol Exp 1972;32:479-94.

${ }^{25}$ Luria AR, Tsvetkova LS. The programming of constructive activity in local brain injuries. Neuropsychologia 1964;2:95-107.

${ }^{26}$ Stern Y. Behavior and the basal ganglia. In: Mayeux R, Rosen W, eds. The Dementias. New York: Raven
Press, 1983:195-209.

${ }^{27}$ Lees AJ, Smith E. Cognitive deficits in the early stages of Parkinson's disease. Brain 1983;106:257-70.

${ }^{28}$ Teuber H-L. Complex functions of basal ganglia. In: Yahr MD, ed. The Basal Ganglia. New York: Raven Press, 1976:151-68.

${ }^{29}$ DeL. Horne DJ. Performance on delayed response tasks by patients with parkinsonism. J Neurol Neurosurg Psychiatry 1971;34:192-4.

${ }^{30}$ Boller F, Passafiume D, Rogers K, Morrow L, Kim Y. Visuospatial impairment in Parkinson's disease: Role of perceptual and motor factors and of disease stage. Neurology (NY) 1982;32:A189. 